\title{
Hybrid Hydrogels Based on Polyethylene Glycol Bioconjugated with Silylated-Amyloidogenic Peptides
}

\author{
Carla C. Decandio, ${ }^{a}$ Sandra V. Vassiliades, ${ }^{a}$ Barbara B. Gerbelli, ${ }^{\circledR a}$ Andrea M. Aguilar ${ }^{b}$ \\ and Wendel A. Alves ${ }^{\circledR} * a$ \\ ${ }^{a}$ Centro de Ciências Naturais e Humanas, Universidade Federal do ABC, \\ 09210-580 Santo André-SP, Brazil \\ ${ }^{b}$ Instituto de Ciências Ambientais, Químicas e Farmacêuticas, Universidade Federal de São Paulo, \\ 09972-270 Diadema-SP, Brazil
}

\begin{abstract}
Hybrid-peptide hydrogel arrangements are a promising alternative to obtaining biocompatible structures at the nanoscopic scale. In this work, a new class of hybrid hydrogels was obtained through the sol-gel process based on the reaction between an amyloid-like octapeptide sequence $[\mathrm{RF}]_{4}$ (where $\mathrm{R}=$ arginine and $\mathrm{F}=$ phenylalanine) covalently bonded with glycine-linked alkoxysilanes, and hybrid silylated polyethylene glycol (PEG). ${ }^{1} \mathrm{H}$ nuclear magnetic resonance (NMR), liquid chromatography-mass spectrometry (LC-MS), and Fourier transform infrared spectroscopy (FTIR) analyses were used, resulting in synthesized precursor/intermediate molecules. The structure of hybrid hydrogel fibers was studied by atomic force microscopy (AFM) and small-angle X-ray scattering (SAXS), where the existence of two regions in the Guinier plot was observed, one being predominantly formed by polymer while the other one by peptide chains. The rheological measurements showed that viscoelastic parameters depend on both the amount of silane peptide in the hydrogel matrix and increased temperature. Also, the FTIR spectra indicated the coexistence of antiparallel and parallel $\beta$-sheet structure patterns into amyloid fibril hydrogels, which can be modulated by peptide-silane coupling in each formulation. The analyzed hydrogels showed thixotropic and shear-thinning rheology at physiological $\mathrm{pH}$, leaving open the opportunity to topical drug delivery system applications in the future.
\end{abstract}

Keywords: peptide materials, polyethylene glycol (PEG), amyloid structures, sol-gel process, rheology, SAXS

\section{Introduction}

In recent years hydrogel materials have been widely investigated for their versatility in biomedicine as tissue engineering scaffolds and controlled release due to their hydrophilic character as well as their biocompatibility. ${ }^{1}$ These systems have a three-dimensional (3D) structure resulting from the self-organization of their constituents. Moreover, another feature of these systems is that they absorb a large amount of water, which allows the diffusion and attachment of molecules and cells. Such applications include tissue engineering, ${ }^{2}$ drug delivery platforms, ${ }^{3}$ and cell culture scaffolds. ${ }^{4}$

The formation of hydrogels can be modulated by either chemical polymerization or physical self-assembly

*e-mail: wendel.alves@ufabc.edu.br of synthetic or natural building component materials. Several of these blocks are macromolecules in a variety of architectures, such as cross-linked polymers, ${ }^{5}$ entangled fibrillar networks, ${ }^{6}$ and colloidal assemblies. ${ }^{7}$ Several research groups are currently combining a variety of molecules to create hybrid supramolecular structures with special properties. Particularly, hydrogels formed by peptides that are chemically linked to polymers have attracted considerable interest because of their favorable viscoelastic properties and specific properties such as biocompatibility, biodegradability, and bioactivity. ${ }^{8}$

Hence, different synthetic strategies have been developed to control secondary structures of self-assembling peptide polymers. The modification of the terminal groups or the side chains on the polypeptide through the incorporation of hydrophobic groups greatly affected their mechanical properties. ${ }^{9}$ The secondary structures also depend on 
the sequence and length of the polypeptide block in the copolymers. Increasing the population of the $\beta$-sheet conformation by chemical modification let to the creation of a more stable hydrogel. ${ }^{10-13}$ The $\beta$-sheet conformation is related to the formation of amyloid $\beta$ (A $\beta)$ protein, which is the cause of Alzheimer's disease. ${ }^{14}$ This secondary structure promotes the aggregation of $\mathrm{A} \beta$ peptide to form fibrils that induce neurotoxicity. ${ }^{15-17}$ Therefore, the $\beta$-sheet conformation, which tends to enhance the hydrophobicity of the polypeptides, is an important factor that may tailor the sol-gel transition as well as the aggregation of polypeptides in an aqueous medium.

Considerable effort has been carried out to develop a correlation between the structural and mechanical properties of the resulting polymer peptide conjugated systems for biomedical applications. ${ }^{18}$ The combination of silicones and peptides to form graft copolymers has been described in previously published studies. ${ }^{19-21}$ More recently, Subra and co-workers ${ }^{21}$ have reported a new strategy based on a mixture of functionalized alkoxysilane-based hybrid materials derived from polyethylene glycol (PEG) and bioactive peptides through a one-step modification under the sol-gel methodology. This attachment opens opportunities to increase the solubility and/or stability of the conjugate into robust nanoscopic structures in solution, so as to obtain hydrogel materials with different viscosities.

Molecular self-assembly of oligopeptides into wellordered nanostructures provides models to explore the development of new materials. ${ }^{14}$ We have recently shown ${ }^{22-24}$ a systematic fibrillation behavior of an alternating $[R F]_{n=1-5}$ peptide (where R: L-arginine, F: L-phenylalanine), which exhibit physicochemical properties of $\beta$-amyloid proteins.

In the present work, we proposed the synthesis of a silylated nonapeptide $\left[\left(\mathrm{CH}_{3} \mathrm{CH}_{2} \mathrm{O}\right)_{3} \mathrm{Si}-\mathrm{G}(\mathrm{RF})_{4}\right]$ (where G: glycine) 1, by using solid-phase peptide synthesis (SPPS) and its conjugation with 3-(triethoxysilyl) propyl isocyanate. At the same time, bifunctional PEG hybrid unit 2 was synthesized by reacting polyethylene glycol (molecular weight $(\mathrm{MW})=4000 \mathrm{Da})$ with 3-isocyanatopropyltriethoxysilane (Scheme 1). Then, the hybrid silylated PEG block was conjugated in a solgel process with hybrid triethoxysilylated peptide $\mathbf{1}$ by combining hydrolysis of ethoxysilyl groups with silanols and their direct condensation to form the hybrid functional silylated PEG-based hydrogels covalently bound to an amyloid-like peptide.

\section{Experimental}

All chemicals had analytical or high-performance liquid chromatography (HPLC) grades and used as received. The peptide was synthesized using a solid phase 9-fluorenylmethoxycarbonyl (Fmoc) strategy. ${ }^{25}$ Protected amino acids (L-Fmoc-Phe-OH, L-Fmoc$\operatorname{Arg}(\mathrm{Pbf})-\mathrm{OH}$ and Fmoc-Gly-OH), Fmoc-Phe-Wang resin (100-200 mesh), 1,3-diisopropylcarbodiimide/ $N$-hydroxybenzotriazole (DIC/HOBt), trifluoroacetic acid (TFA), anisole, thioanisole, 1,2-ethanedithiol (EDT), dichloromethane (DCM), dimethylformamide (DMF), 1-methyl-2-purrolidinone (NMP), and tetrahydrofuran (THF) were purchased from Sigma-Aldrich (Saint Louis, MO, USA). In the latter, the THF solvent was distilled under nitrogen from $\mathrm{Na} / \mathrm{K}$.

Peptide-silane synthesis $\left[\left(\mathrm{CH}_{3} \mathrm{CH}_{2} \mathrm{O}\right)_{3} \mathrm{Si}-\mathrm{G}[\mathrm{RF}]_{4}\right]$ (1)

After hydration of the Fmoc-Phe-Wang resin with dichloromethane for $15 \mathrm{~min}$, the Fmoc protecting group was removed in two cycles by basic hydrolysis with a 4-methylpiperidine in DMF (20\%, $10 \mathrm{~mL}, 15 \mathrm{~min})$. Coupling was carried out with 2.50 equivalent of amino acid and 5.00 equivalent DIC and HOBt in NMP (10 mL) in orbital shaking for 4 and $12 \mathrm{~h}$ (Phe/Gly and Arg, respectively). All reaction cycles were monitored by the Kaiser ninhydrin test. ${ }^{26}$ After the Gly coupling, the peptide was silylated with 3-(triethoxysilyl)propyl isocyanate $(3)(0.52 \mathrm{~g}, 0.50 \mathrm{~mL}, 2.10 \mathrm{mmol})$ in anhydrous DMF $(10 \mathrm{~mL})$ in the presence of diisopropylethylamine (0.39 g, $0.50 \mathrm{~mL}, 3.00 \mathrm{mmol}$ ) overnight. ${ }^{19,20}$ The hybrid peptide was deprotected and released from the resin under acid hydrolysis conditions with a mixture of TFA $(90.0 \%)$, thioanisole (5.00\%), 1,2-ethanedithiol (3.00\%), and anisole $(2.00 \%)$. The reaction was left in orbital agitation for $4 \mathrm{~h}$, the supernatant was collected by vacuum filtration, and ice-cold diethyl ether was added to precipitate the silylated peptide, which was successively washed with ice-cold diethyl and then lyophilized. Product $\mathbf{1}$ was isolated as a white powder without further purification.

PEG-(bis)silylated synthesis $\left[\left(\mathrm{C}_{2} \mathrm{H}_{5} \mathrm{O}\right)_{3} \mathrm{Si}-\mathrm{PEG}-\mathrm{Si}\left(\mathrm{C}_{2} \mathrm{H}_{5} \mathrm{O}\right)_{3}\right]$ (2)

Polyethylene glycol 4000 (4.0 g, $1 \mathrm{mmol}$ ) was vacuum dried for $24 \mathrm{~h}$ at $80{ }^{\circ} \mathrm{C}$ and dissolved in anhydrous tetrahydrofuran $(12 \mathrm{~mL})$ under nitrogen atmosphere. Then triethylamine $(1.21 \mathrm{~g}, 1.66 \mathrm{~mL}, 12 \mathrm{mmol})$ and $3(0.74 \mathrm{~g}$, $0.74 \mathrm{~mL}, 2.99 \mathrm{mmol}$ ) were added to the solution and the mixture was refluxed for $48 \mathrm{~h}$. The solvent was removed under reduced pressure, and the crude residue was washed several times with hexane and then vacuum dried. Product 2 was obtained as a white powder without further purification. Hybrid PEG was then stored at $4{ }^{\circ} \mathrm{C}$ under nitrogen. 
(a) Synthesis of silanized peptide

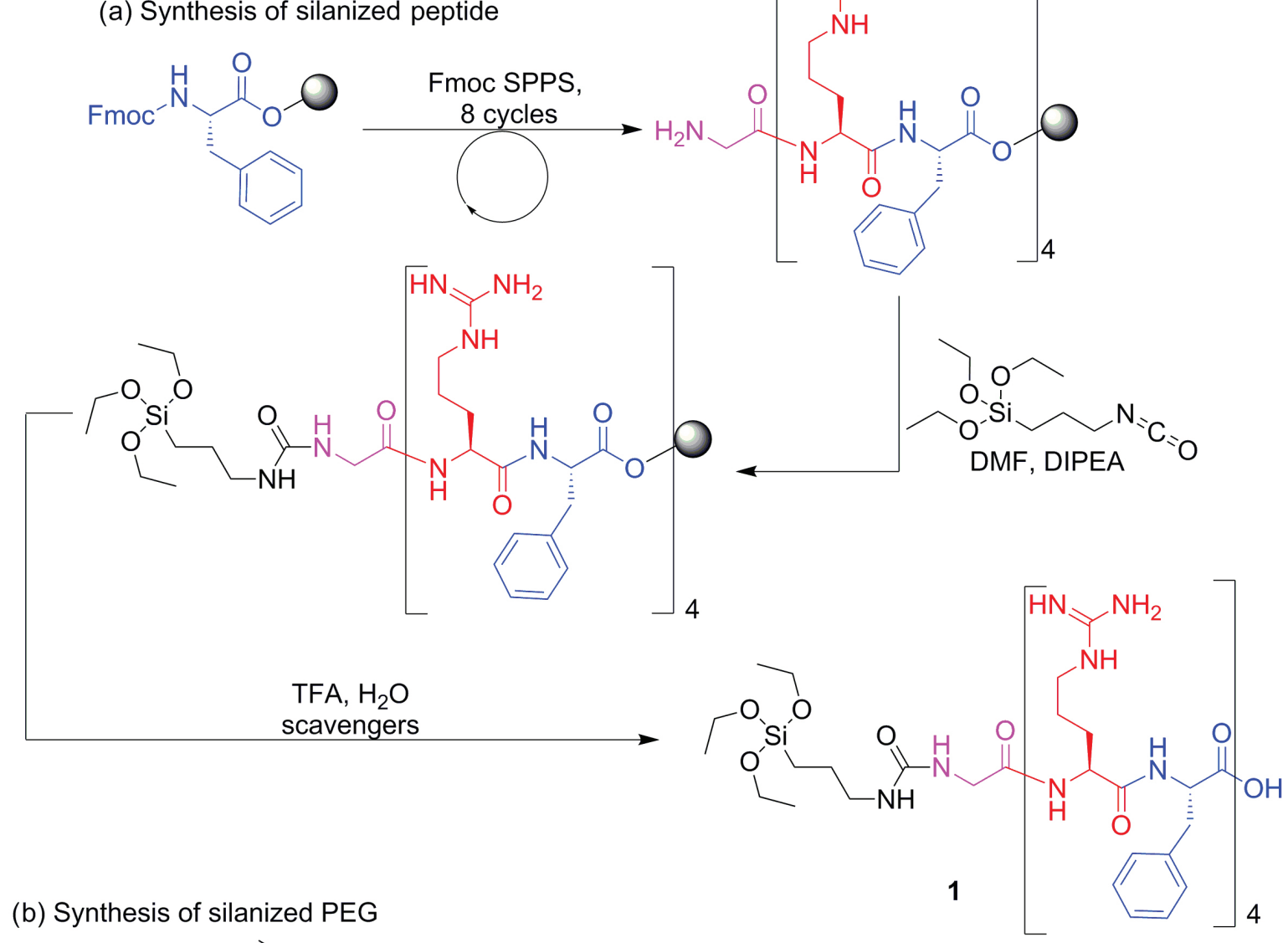<smiles>CCOC(C)(CCN(C)CC)COC</smiles><smiles>CCO[Si](CCCNC(=O)COCC(C)OC(=O)NCCC[Si](OCC)(OCC)OCC)(OCC)OCC</smiles>

(c) Hydrogel preparation
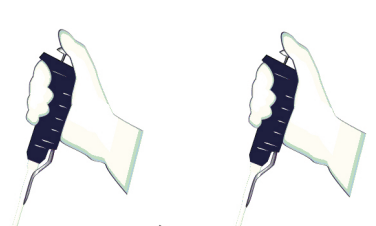

2

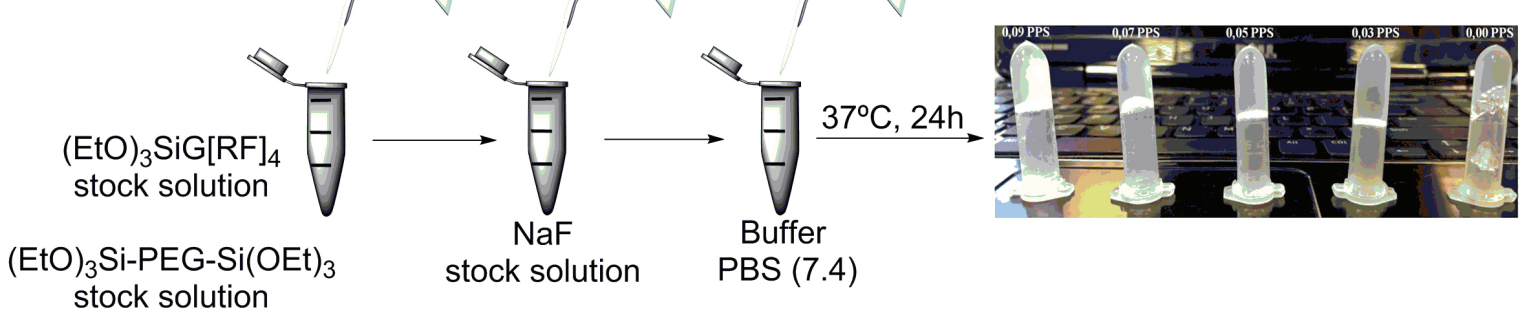

Scheme 1. The general route of synthesis of silylated precursors and hybrid hydrogel. (a) Synthesis of compound $\mathbf{1}$ by SPPS; (b) synthesis of compound $\mathbf{2}$; (c) preparation of hybrid hydrogel.

${ }^{1} \mathrm{H}$ NMR (500 MHz, $\mathrm{CDCl}_{3}$ ) $\delta 5.00$ (br s, 2H, NH), 3.80 (q, $12 \mathrm{H}, J 7.0 \mathrm{~Hz}, \mathrm{C}_{2} \mathrm{CH}_{3}$ ), 3.49 (s, $499 \mathrm{H}, \mathrm{CH}_{2}-\mathrm{PEG}$ ), $3.15\left(\mathrm{dd}, 4 \mathrm{H}, J 12.4,6.8 \mathrm{~Hz}, \mathrm{C}_{2} \mathrm{NH}\right), 1.59$ (q, $4 \mathrm{H}, J 7.6$ $\left.\mathrm{Hz}, \mathrm{CH}_{2} \underline{\mathrm{C}}_{2} \mathrm{CH}_{2}\right), 1.21$ (t, $\left.18 \mathrm{H}, J 7.0 \mathrm{~Hz}, \mathrm{CH}_{2} \underline{\mathrm{CH}}_{3}\right), 0.63-$ $0.58\left(\mathrm{~m}, 4 \mathrm{H}, \mathrm{CH}_{2} \mathrm{CH}_{2} \mathrm{CH}_{2}\right.$ ).

\section{Preparation of hybrid hydrogel samples}

The hybrid hydrogels were prepared in a mixture of individual solutions. Different concentrations of $\mathbf{1}$ were prepared in the range of 0.2 to $0.8 \mathrm{wt} . \%$ in a dimethyl 
sulfoxide (DMSO) and Dubelcco's phosphate buffer (DPBS, pH 7.4) solution in the ratio of $1 / 9(\mathrm{v} / \mathrm{v})$ and sonicated until the solutions were clear. For each prepared sample, the silylated-PEG 2 was added to correspond to a concentration of $9.5 \mathrm{wt} . \%$ in the final volume of $1 \mathrm{~mL}$, as shown in Table 1. In all samples, $200 \mu \mathrm{L}$ of $\mathrm{NaF}$ (2.5 wt.\%) in DPBS solution were added as a catalyst for the reaction. After complete solubilization, the samples were heattreated for $24 \mathrm{~h}$, first in a water bath at $37{ }^{\circ} \mathrm{C}$, and then refrigerated at $4{ }^{\circ} \mathrm{C}$. An inverted tube test was performed to observe hydrogel formation (see Scheme 1).

Table 1. Composition and nomenclature of all hybrid hydrogels used in this work

\begin{tabular}{lcc}
\hline $\begin{array}{l}\text { Hydrogel / (mol\% of } \\
\text { 1 regarding hybrid } \\
\text { block 2) }\end{array}$ & [1] / wt.\% & [2] / wt.\% \\
\hline 11 & 0.8 & 9.5 \\
7.0 & 0.7 & 9.5 \\
4.0 & 0.5 & 9.5 \\
2.0 & 0.3 & 9.5 \\
0.5 & 0.2 & 9.5 \\
Non-charged & 0 & 9.5 \\
\hline
\end{tabular}

\section{Characterization techniques}

\section{Spectroscopic characterization}

Fourier transform infrared spectroscopy (FTIR) assays were carried out on a PerkinElmer Spectrum Two spectrometer equipped with a universal attenuated total reflection (UATR) accessory for solid samples at the Multiuser Central Facilities (UFABC, Brazil), and spectra were measured at a resolution of $2 \mathrm{~cm}^{-1}$. Data were collected with previously lyophilized samples placed in contact with the diamond crystal. Spectra were obtained from 64 scans in the range $500-4000 \mathrm{~cm}^{-1}$. Spectra were averaged and the background was subtracted; resonances were deconvoluted and fitted to Gaussian profiles using the Fityk program ${ }^{27}$ to enable accurate assignment.

${ }^{1} \mathrm{H}$ nuclear magnetic resonance (NMR) spectra were recorded at room temperature in deuterated solvents on a Varian 500 spectrometer operating at $500 \mathrm{MHz}$ in the Multiuser Central Facilities (UFABC, Brazil). Chemical shifts $(\delta)$ were reported in parts per million using residual non-deuterated solvents as internal references $\left(\mathrm{CHCl}_{3}\right.$ in $\mathrm{CDCl}_{3}, \delta \mathrm{H}=7.26 \mathrm{ppm}$ ). Signals are indicated as $\mathrm{s}$ (singlet), d (doublet), $\mathrm{t}$ (triplet), q (quartet), dt (double triplet), $\mathrm{m}$ (multiplet), br (broad). Coupling constants are measured in hertz.

\section{Rheological analysis}

Viscoelastic properties of all hydrogels were analyzed using a controlled-stress Malvern rheometer (Kinexus lab+) equipped with a parallel plate geometry and a sample gap of $0.1 \mathrm{~mm}$ at $25^{\circ} \mathrm{C}$. All measurements were performed in triplicate. Oscillatory analyses were conducted at a constant frequency of $1 \mathrm{~Hz}$ and a stress sweep from 0.1 to $200 \mathrm{~Pa}$ to determine the viscoelastic region of the sample. A constant shear stress of $1 \mathrm{~Pa}$ was later selected to perform the frequency sweep from 0.1 to $100 \mathrm{~Hz}$, which was within the previously determined linear viscoelastic region for all samples, and the storage $\left(G^{\prime}\right)$ and loss (G") moduli were recorded. For the determination of the gel strength (S) and viscoelastic exponent (n), also called the relaxation exponent, the power-law model was used in equation 1 . The variation of the storage modulus (G') at low frequencies in a log-log plot of G" versus frequency follows the power law equation as presented in equation 1 :

$\mathrm{G}^{\prime}=\mathrm{S} \omega^{\mathrm{n}}$

where $\mathrm{G}^{\prime}$ is the storage modulus, $\mathrm{S}$ is the hydrogel strength, $\omega$ is the frequency, and $n$ is the viscoelastic exponent. The $\mathrm{n}$ and $\mathrm{S}$ values allowed the quantitative estimation of the strength of hydrogel structures that can be related to the crosslinking density within the polymer network. Continuous testing to analyze the thixotropic properties of the materials was performed using a controlled shear rate procedure in the range from 0.1 to $100 \mathrm{~s}^{-1}$, each stage lasting $2 \mathrm{~min}$.

\section{Small-angle X-ray scattering (SAXS)}

Small-angle X-ray scattering (SAXS) experiments were carried out at the Brazilian Nanotechnology National Laboratory (Campinas, Brazil) by using an X-ray wavelength of $1.54 \AA$ and Pilatus $300 \mathrm{~K}$ detector (Dectris). The sample-detector distance used was $1.5 \mathrm{~m}$, leading to a range that was experimentally accessible $0.015<\mathrm{q}<0.31 \AA^{-1}$, wich the modulus $\mathrm{q}=(4 \pi \operatorname{sen}(2 \theta) / \lambda)$ of the transfer moment vector, where $\lambda$ is the radiation wavelength and $2 \theta$ is the scattering angle. The hydrogel samples were placed in hermetically sealed metal supports with mica windows that allow the experiments to be carried out by transmission. Ten frames of $60 \mathrm{~s}$ each were recorded during the flow to avoid radiation damage and afterward, we calculated their average before background subtraction. All data treatment was carried out by Fit2D software, ${ }^{28}$ and the inverse Fourier transform (IFT) analyses were performed by the SUPERSAXS package. ${ }^{29}$ 


\section{Microscopic measurements}

The gels were lyophilized, deposited on a silicon-plate surface, and exposed to scanning electron microscopy (SEM) analysis. Sixty seconds of deposition and $40 \mathrm{~mA}$ current were used for metallization. The scanning electron of the hybrid materials was obtained by a highresolution JSM 6330F instrument (Inspect F50). Secondary backscattered electrons were collected from Au-coated samples impinged by electron beams with an energy of $3 \mathrm{kV}$. The MultiMode VII Bruker NanoScope V was used for atomic force microscopy (AFM). The system was operated in peak-force mode using a silicon tip with constant $0.4 \mathrm{~N} \mathrm{~m}^{-1}$ nominal force and frequency of $2 \mathrm{kHz}$. The gels in solution were deposited on mica substrates. Images with typical $512 \times 512$-pixel sizes were obtained, covering areas of $1 \times 1 \mu \mathrm{m}$ to $5 \times 5 \mu \mathrm{m}$. Both microscopy equipment and experimental materials (SEM and AFM) are located at the Brazilian Nanotechnology National Laboratory (Campinas, Brazil).

\section{Results and Discussion}

The monofunctional nonapeptide $\mathbf{1}$ was synthesized by SPPS strategy via Fmoc procedure..$^{30,31}$ This approach allows the preparation of interesting compounds in a one-pot reaction, leading to higher yield and purity when it is compared with liquid phase peptide synthesis (see Scheme 1a). The functionalization of the peptide with the 3-(triethoxysilyl) propyl isocyanate (3) was performed as the last step of SPPS, using $N, N$-diisopropylethylamine (DIEA) as a proton abstraction base. The nucleophilic attack of the nitrogen electron pairs on the cyanate carbon leads to carbamate formation after a rearrangement of bonds $(\mathrm{N}=\mathrm{C}=\mathrm{O} \rightarrow \mathrm{NHC}=\mathrm{ONH})$. After the cleavage step, the silylated peptide was recrystallization-isolated and characterized by liquid chromatography-mass spectrometry (LC-MS). The mass spectrum (Figure S1, Supplementary Information (SI) section) has an $m / z 1537$ peak, which is attributed to the molecular ion $[\mathrm{M}+\mathrm{H}]^{+}$, indicating the presence of the silylated group in the peptide chain (theoretical mass of $1536 \mathrm{~g} \mathrm{~mol}^{-1}$ ). Another attributed fragment is the $m / z 1185$ peak, which is related to the loss of $(\mathrm{EtO})_{3} \mathrm{SiG}$ group and carboxylic acid $\left(\left[\mathrm{M}-\mathrm{C}_{12} \mathrm{H}_{26} \mathrm{~N}_{2} \mathrm{O}_{5} \mathrm{Si}\right]^{+}\right.$ and $\left[\mathrm{M}-\mathrm{CO}_{2} \mathrm{H}\right]^{+}$, respectively). The $m / z, 1013$ peak refers to protonated peptide fragment after loss of silylated $\left(\left[\mathrm{M}-(\mathrm{EtO})_{3} \mathrm{SiC}_{3} \mathrm{H}_{6}\right]^{+}\right)$and dipeptide $\mathrm{RF}\left(\left[\mathrm{M}-\mathrm{C}_{15} \mathrm{H}_{22} \mathrm{~N}_{5} \mathrm{O}_{3}\right]^{+}\right)$. The $m / z 247$ fragment was attributed to the carbamate group breakdown $\left(\left[\mathrm{M}-\mathrm{C}_{10} \mathrm{H}_{23} \mathrm{NO}_{4} \mathrm{Si}\right]^{+}\right)$.

Bifunctional $\mathrm{PEG}_{4000} 2$ was prepared by nucleophilic reaction between PEG hydroxyl and the cyanate group carbon, as discussed above (Scheme 1b). Initially, PEG $_{4000}$ was dried in a vacuum oven to remove any probable moisture present in the compound, decreasing the likelihood of byproduct formation due to the presence of water. The reaction between dry-PEG and $\mathbf{3}$ was carried out in dry THF under $\mathrm{N}_{2}$ and reflux for $48 \mathrm{~h}$. Then, product 2 was recrystallization-isolated after several hexane washings, and the final compound was dried in a vacuum oven and characterized by NMR (Figure S2, SI section). In the proton spectrum of $\mathbf{2}$, the presence of a triplet and a quartet with a chemical shift of 1.21 and $3.80 \mathrm{ppm}$ were respectively attributed to the coupling between $\mathrm{CH}_{3}-\mathrm{CH}_{2}$ and $\mathrm{CH}_{2}-\mathrm{CH}_{3}$ of the ethoxy, while 4.95-5.08 ppm shows a multiplet that can be attributed to protons bound to carbamate nitrogen, proving the effectiveness of the functionalization performed.

The hybrid hydrogel composed of $\mathbf{1}$ and $\mathbf{2}$ was prepared in a DPBS-buffer solution in a self-assembling sol-gel process consisting of hydrolysis of silane groups followed by condensation into siloxanes ( $\mathrm{SiOH} \rightarrow \mathrm{Si}-\mathrm{O}-\mathrm{Si}$ ), using a sodium fluoride solution as the hydrolysis agent of the silylated groups present in $\mathbf{1}$ and $\mathbf{2}$. The concentration of PEG-silylated was kept constant (9.5 wt.\%), and 6 samples were prepared with different concentrations of silylated peptides, which were heated for $24 \mathrm{~h}$ at $37^{\circ} \mathrm{C}$, and then kept refrigerated for $24 \mathrm{~h}$ at $4{ }^{\circ} \mathrm{C}$ (see Table 1).

In order to investigate mechanical properties, we performed an oscillatory analysis of the hybrid hydrogels containing different quantities of peptides in the matrix of PEG-silane, where the systems are characterized by storage $\left(G^{\prime}\right)$ and loss (G') moduli as an oscillation frequency function (Figure 1a).

The G' behavior for the bare PEG-Si 2 hydrogel is practically constant up to $8 \mathrm{rad} \mathrm{s}^{-1}$ and grows quickly to higher frequencies, and with the addition of the peptide to the hydrogel, the behavior becomes practically linear. It is an indicative that the presence of the peptide in the hydrogel promotes greater mechanical stability. Besides, when increasing the amount of peptides in the system, the G' values become an order of magnitude that is higher than the non-charged polymer networks, showing that gel mixtures are stronger than polymer-only gels. We also observed that the G" values for non-charged hydrogels present sinusoidal behavior that disappears with the incorporation of peptides.

All the samples present solid-like properties with G' parameters higher than G' ones (Figures 1a-1f). We also noticed an increased dependence of $G^{\prime}$ values when increasing the concentration of peptides in the hydrogel, which results into greater cross-linking among polymer chains.

Data obtained from frequency measurements for all hybrid hydrogels are summarized in Figure 1, where G' values are given as a function of peptide concentrations, 

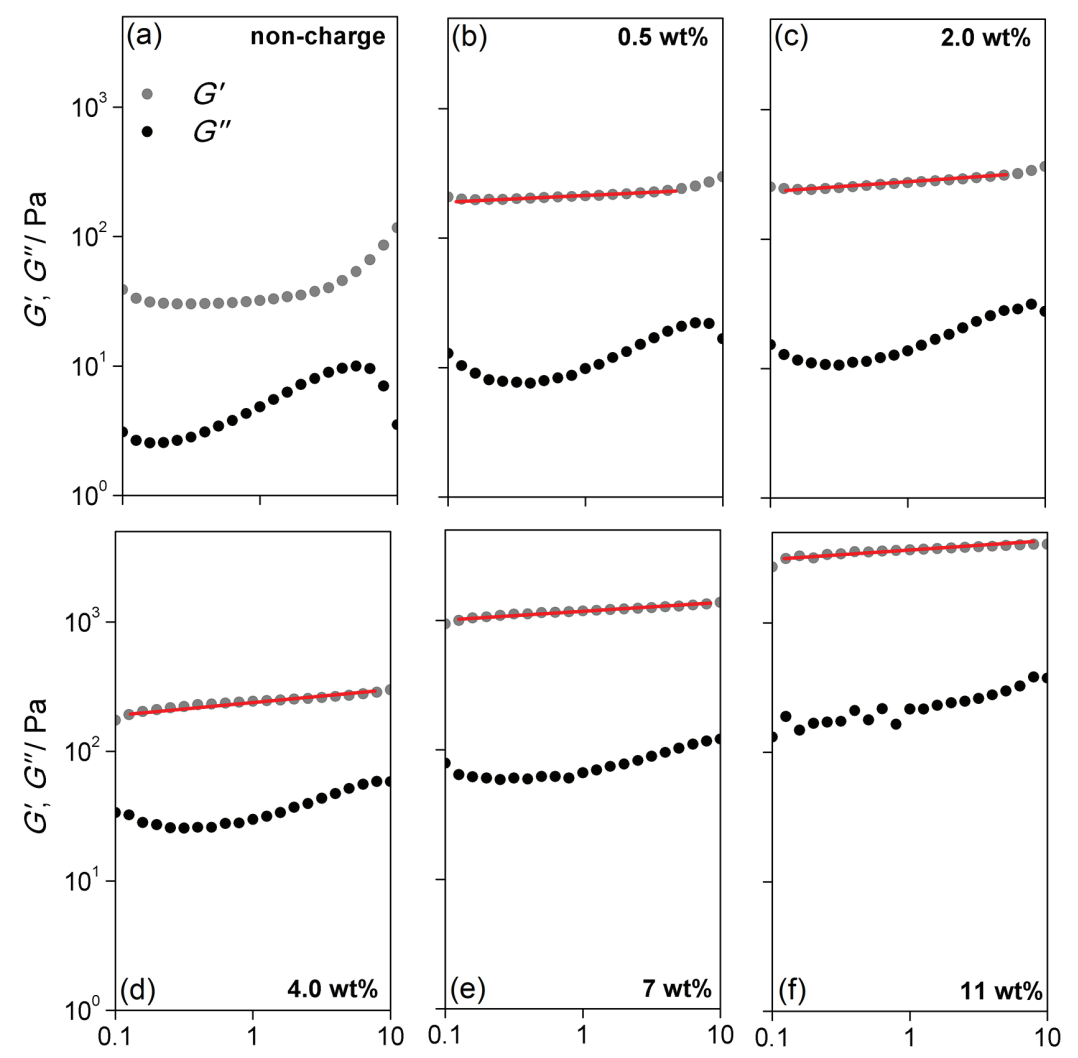

$\omega / \mathrm{rad} \mathrm{s}^{-1}$

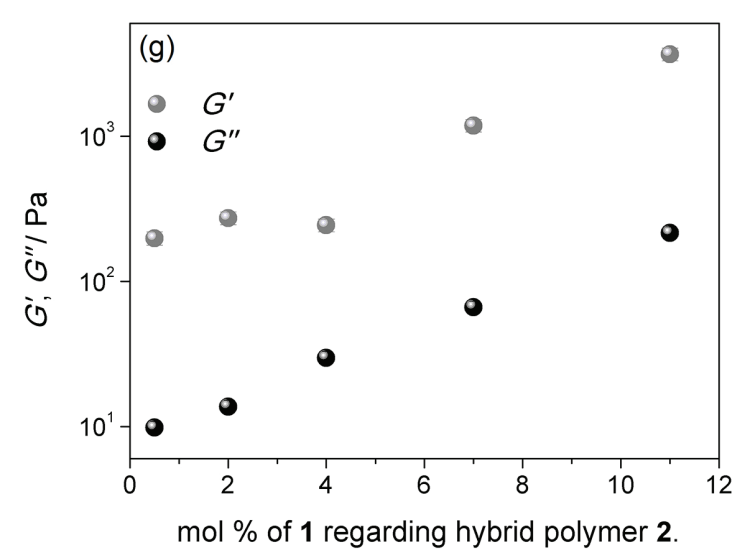

Figure 1. Elastic (G', gray dots) and viscoelastic (G', black dots) parameters as a function of frequency for hybrid hydrogels. The red line shows the adjustment of the experimental curves using the power-law model and G' and G" behaviors, which depend on the increasing peptide concentrations in the hydrogel.

and we notice two G' types of behavior: the first region did not present any change for G' values up to the moment the peptide concentration reached $4 \mathrm{wt} . \%$. On the other hand, when the concentration reaches higher levels, G' values start increasing, indicating that the viscoelasticity properties of hydrogels can be controlled, depending on the increasing peptide concentration in the composite.

The $\mathrm{n}$ and $\mathrm{S}$ parameters were calculated using equation 1 , which yield a quantitative estimation of how strong the hydrogel structures are, which can be related to the crosslinking density within the polymer network. The proposed model is agreeable, except for the non-charged polymer $\mathbf{2}$. In this case, with increasing concentrations of the hybrid peptide-silane compound $\mathbf{1}$ in the hydrogels, higher values for $G$ ' and $S$ were observed, despite the decrease in the $n$ exponent values (Table 2), indicating that the insertion of the peptide concentration into the polymer hydrogel matrix improves the inter- and intramolecular interactions network along the polymer chain. The $\mathrm{n}$-parameters for all hydrogels prepared in this work were less than 0.11 ( $\mathrm{n}$ values range from 0 to 1 ), indicating a non-Newtonian shear-thinning flow behavior. 
Table 2. Coefficient determination for the storage modulus (G'), gel strength (S), and viscoelastic exponent (n) of the hydrogels obtained

\begin{tabular}{lccc}
\hline $\begin{array}{l}\text { Hydrogel / (mol\% } \\
\text { of } \mathbf{1} \text { regarding } \\
\text { hybrid block 2) }\end{array}$ & $\mathrm{G}^{\prime} / \mathrm{kPa}$ & $\mathrm{S} / \mathrm{kPa}$ & $\mathrm{n}$ \\
\hline 11 & $3.6 \pm 0.1$ & $3.28 \pm 0.39$ & $0.062 \pm 0.005$ \\
7.0 & $1.2 \pm 0.1$ & $1.04 \pm 0.07$ & $0.071 \pm 0.003$ \\
4.0 & $2.4 \pm 0.2$ & $0.48 \pm 0.03$ & $0.074 \pm 0.002$ \\
2.0 & $2.7 \pm 0.1$ & $0.23 \pm 0.03$ & $0.087 \pm 0.005$ \\
0.5 & $2.1 \pm 0.1$ & $0.19 \pm 0.02$ & $0.041 \pm 0.004$ \\
\hline
\end{tabular}

It is already known that PEG not only is sensitive to thermal changes but also has lower mechanical properties when the temperature is increased. ${ }^{32,33}$ For instance, a few rheology experiments were carried out in the same way as described before with non-charge polymers $\mathbf{2}$, and two types of composite systems, 4 and $11 \mathrm{wt} . \%$, in the $25-60{ }^{\circ} \mathrm{C}$ temperature range (Figure 2). In this case, for all samples, the storage modulus ( $G^{\prime}$ ) was greater than the loss modulus (G') of the hydrogels over the studied temperature range, indicating that the viscoelastic behavior of the gels is independent of the temperature gradient. However, for noncharged hydrogel 2, G' and G" values fell until temperature reached $38{ }^{\circ} \mathrm{C}$ and started rising after that.

When incorporating the amyloid-peptide into the composite (4.0 and 11 wt.\%), G' behavior changed completely, as the value of $\mathrm{G}^{\prime}$ is an order of magnitude higher when compared with the non-charged PEG-Si $\mathbf{2}$, resulting in a much stronger gel in all the studied temperature range. Moreover, there was no decrease of G' values as the temperature increased. This behavior is associated with the molecular mobility of polymer chains in the temperature range investigated, leading to an increase in the cross-linking density and mechanical stability of the material.
Studies in the literature ${ }^{12}$ have demonstrated that the presence of PEG can affect the secondary structures of peptides and as a consequence promote changes in the mechanical properties of materials. To evaluate possible changes in the secondary structure of peptides in the hydrogels, FTIR measurements were performed by using different relationships between $\mathbf{1}$ and $\mathbf{2}$. We have focused our analyses on the amide I region (Figure 3a), and the complete spectrum with some indexations from the main bands are present in the Supplementary Information section (Figure S3). In Figure 3a, the red line represents the spectrum of compound $\mathbf{1}$, where we observed two bands, the first one in the position around $1630 \mathrm{~cm}^{-1}$ assigned to antiparallel $\beta$-sheets, which can be related to stretching of bonds between adjacent strand layers, ${ }^{12,22}$ and the second band around $1661 \mathrm{~cm}^{-1}$ correspondent to TFA counterions. ${ }^{13}$ The hybrid hydrogels are depicted by a pronounced band of around $1690 \mathrm{~cm}^{-1}$, which can be associated to secondary parallel $\beta$-sheet structures. However, such band is not present in the neat self-assembled $\left[\mathrm{RF}_{4}\right.$ peptide compound. ${ }^{22,24}$

The secondary structures of hydrogels were quantified by band fitting deconvolution (see Figure S4, SI section). Here we used only four Gaussian to adjust the data: two of them related to secondary structures (parallel and antiparallel $\beta$-sheets), and the other two attributed to the TFA ion and $\mathrm{C}=\mathrm{O}$ stretching vibrations from carbonyl and carboxylic groups. ${ }^{34,35}$

In Figure 3b, we plotted the behavior of $\beta$-sheet populations as a function of relationship of $\mathbf{1}$ and $\mathbf{2}$ components in the hydrogels. We can clearly note the presence of both $\beta$-sheet structures (parallel and antiparallel orientation of strands) in all samples.

The neat self-assembled peptides' $[\mathrm{RF}]_{4}$ contribution is related to antiparallel $\beta$-sheets and a small population of parallel secondary structures. ${ }^{22}$ However, the population of parallel strands of the hybrid hydrogel becomes dominant
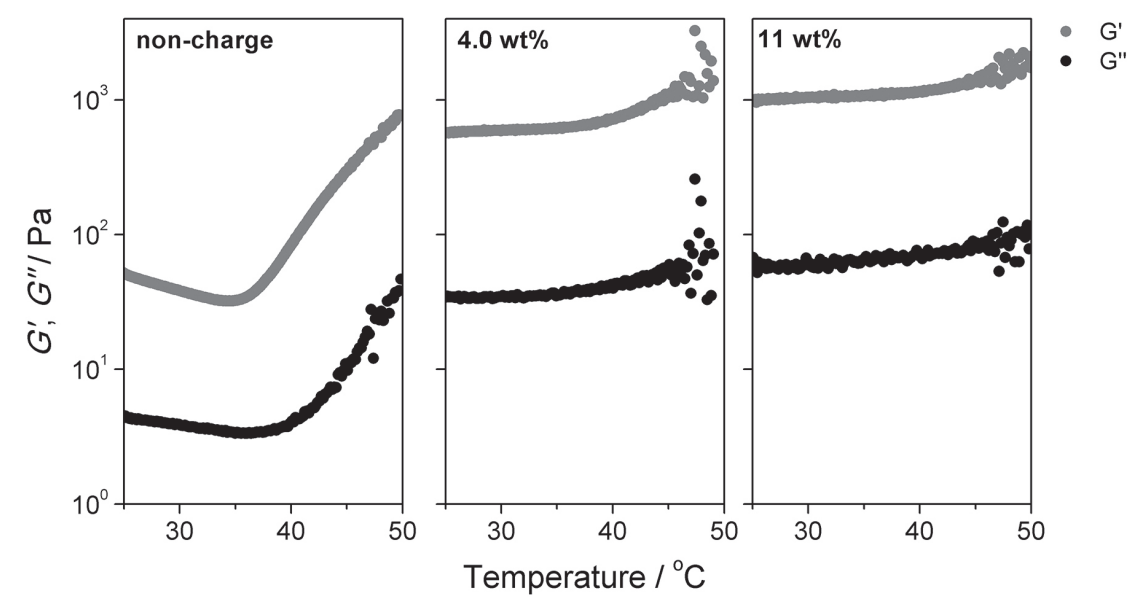

Figure 2. G' (gray dots) and G" (black dots) parameters according to temperature variation for non-charged polymer 2 and composites in 4 and 11 wt.\%. 

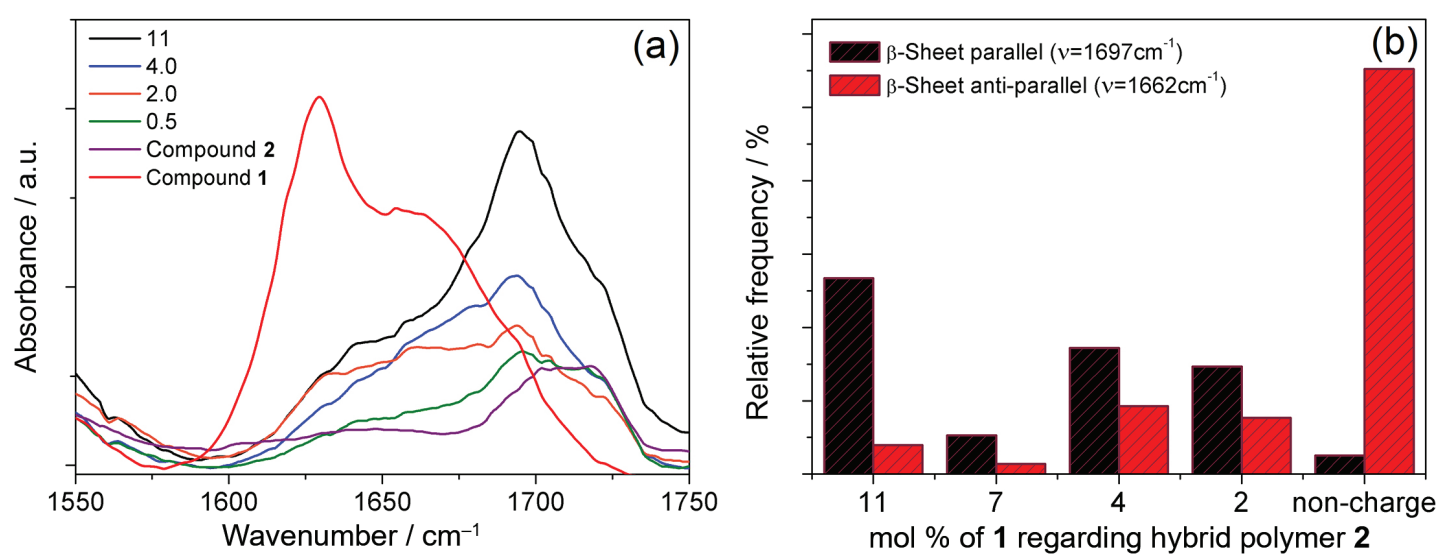

Figure 3. Secondary structure assays (a) FTIR data from hybrid hydrogels and (b) frequency of population of $\beta$-sheets due to the mol\% of $\mathbf{1}$ regarding hybrid polymer 2 .

while the antiparallel alignment decreases. The hydrogel with the highest amount of peptides (black column) predominantly promotes the parallel $\beta$-sheets (Figure $3 b$ ). This leads to the belief that the presence of the silane groups in both structures $\mathbf{1}$ and $\mathbf{2}$ promotes this peptidechain reorganization in the aggregated phase, induced by the sol-gel process during hydrogel production. Besides, when increasing the amount of peptides in the matrix, the intensity of the peak in $1700 \mathrm{~cm}^{-1}$ increased as well, which may be related to the better structural organization within composite networks, as shown in Figure $3 \mathrm{~b}$.

Studies have proven that peptide hydrogels that feature mostly phenylalanine residues have shown higher viscoelastic modulus values. Such higher values are due to the interaction among aromatic-aromatic side-chain amino acids that favor the conversion of the peptides into amyloid-like fibrils, which increases the storage modulus of hydrogels, ${ }^{13}$ indicating that secondary structures play a fundamental role in the mechanical properties in this study. In fact, in a previously published work, Besenius and co-workers ${ }^{10}$ identified that the conversion of antiparallel to parallel $\beta$-sheet structures within the peptide motif attached to PEG termini promotes changes in the mechanical properties of the hydrogel. For the hydrogels with parallel $\beta$-sheet assemblies, they observed the same viscoelastic behavior that was obtained in this work, which also modulated this transition due to the presence of the silane group that induced the self-assembly of the peptides in parallel $\beta$-sheet structures.

SAXS experiments were performed so as to obtain information from the supramolecular organization of hybrid hydrogels. Figure S5 (SI section) shows the scattered intensity curves $\mathrm{I}(\mathrm{q})$ as a result of the scatter difference vector $\mathrm{q}$ for the precursor compounds as well as for the hybrid hydrogels.

For the compound $\mathbf{2}$ scattering curve, we employed the IFT, ${ }^{36,37}$ which provides the function of pair-distance distribution $\mathrm{p}(\mathrm{r})$ and allows particle size and shape determination in real space. The polymeric behavior of the $\mathrm{p}(\mathrm{r})$ characteristic was observed in these systems, ${ }^{24}$ as shown in Figure S5b (SI section). This same treatment cannot be applied to sample $\mathbf{1}$, as it presents a structure factor in the lower angle region. Thus, the generalized method of the inverse Fourier transform (GIFT) was adopted. Besides the form factor information above described, the fractal-mass structure was added to the experimental curve in the low angle region (Figure S5a, SI section), which fell by $\mathrm{q}^{-2.6}$, a feature of two-dimensional fractal masses. ${ }^{38}$ The $[R F]_{4}$ system has been widely studied, proving that it features the formation of cylinders above the cac (critical aggregation concentration). ${ }^{22-24}$ Such behavior was also observed for compound $\mathbf{1}$, demonstrating that the addition of the silane group and the glycine residues did not interfere in the system morphology (Figure S5c, SI section). The cylinder radius can be estimated using the $\mathrm{p}(\mathrm{r})$ curve, which corresponds to the inflection point of the curve at its maximum peak. In this case, a radius of ca. $4.3 \mathrm{~nm}$ was obtained, a value that is close to those reported previously in the literature. ${ }^{22}$

The $I(q) \times q$ curve behavior of the hybrid systems (Figure 4) demonstrated an increase in the curve ground at the lower angle region, which can be related to the addition of $\mathbf{1}$ in the hydrogel matrix. Such result indicated not only the formation of larger aggregates but also the contribution of the structure factor increases, which is associated to only-peptide behavior. Besides, a diffuse peak around $0.05 \mathrm{~nm}^{-1}$ was observed in all the curves, corresponding to the polymeric scattering curve decay. Thus, the adjustment fitting of the experimental data was adopted as a sum model of the scattering intensities $\mathrm{I}_{\mathrm{FIT}}=\mathrm{S}\left[\mathrm{A} \mathrm{I}_{\text {Peptide }}(\mathrm{q})+\mathrm{B} \mathrm{I}_{\text {Polymer }}(\mathrm{q})\right]$, where $\mathrm{I}_{\text {Peptide }}(\mathrm{q})$ and $I_{\text {Polymer }}(q)$ represent the scattering behavior of compounds $\mathbf{1}$ and $\mathbf{2}$, respectively, $\mathrm{S}$ is a scale factor, and $\mathrm{A}$ and $\mathrm{B}$ are corresponding proportion factors. 

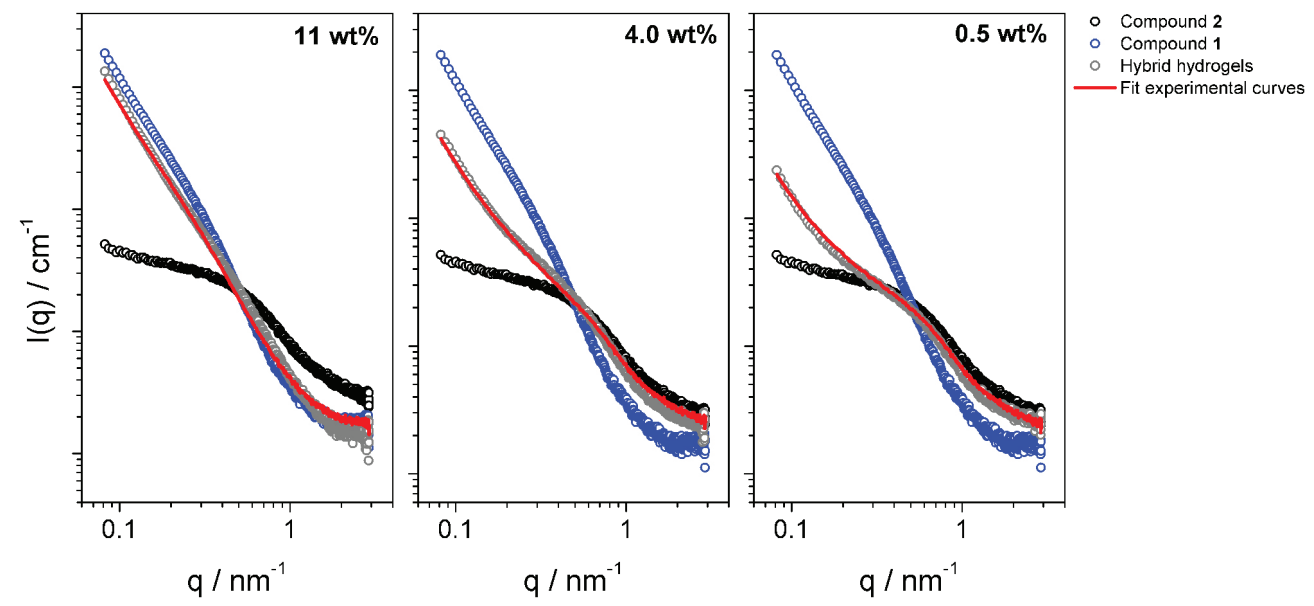

Figure 4. Intensity as a result of the hybrid hydrogel q vector (gray dots) with different peptide concentrations. The blue and black dots are the data of compounds $\mathbf{1}$ and $\mathbf{2}$, and the red line is the fit of experimental data.

The fittings with the proposed model are depicted in Figure 4. This model fits the experimental data very well, validating that the hybrid hydrogel system is composed of two distinct and independent regions, where one is longcylinder peptides and the other one is a polymer-chainrelated fractal component.

Table 3 shows the values of parameters A and B obtained when adjusting the SAXS data, shown in Figure 4 . When keeping the polymer ratio (B) value constant, parameter (A) decreases as expected. This was an experimentally controlled parameter to obtain hydrogels (Table 1) and observe the same behavior, which gives even greater credibility to the proposed model.

Table 3. Parameters A and B used to fit the SAXS data of hydrogels

\begin{tabular}{lll}
\hline $\begin{array}{l}\text { Hydrogel / (mol\% } \\
\text { of regarding } \\
\text { hybrid block 2) }\end{array}$ & A & B \\
\hline 11 & 2.40 & 1 \\
7.0 & 0.50 & 1 \\
4.0 & 0.25 & 1 \\
2.0 & 0.20 & 1 \\
0.5 & 0.12 & 1 \\
\hline
\end{tabular}

Atomic force microscopy experiments were carried out in order to observe the direct space of the hydrogels' morphological structures. Figures 5a-5b correspond to the $\mathbf{1}$ and $\mathbf{2}$ precursor systems, respectively. Images of compound $\mathbf{1}$ show the formation of fibrillar structures in the nanoscopic scale, with homogeneous distribution and formation of sinuous structures, suggesting high fiber flexibility. The Gwyddion program ${ }^{39}$ was used to estimate the average fiber diameter, which in this case was $15.6 \mathrm{~nm}$ (Figure 5a). Such value is comparable with the values determined by
Alves and co-workers. ${ }^{22}$ Another interesting feature was the interconnection of the fibers, which formed an entangled network that is extremely important in gel formation. The characteristics found for the silane peptide are similar to only $[\mathrm{RF}]_{4}$ compound materials, ${ }^{22-24}$ suggesting that the silane group retains the characteristic of conformation-rich fibrillar structures. An amorphous structure extent in all the images of compound $\mathbf{2}$ was detected, which is a feature of synthetic polymers (Figure 5b). Two non-homogeneous distribution for hydrogel regimes (4 wt.\%, Figures 5c-5d) were observed: an amorphous one and a fibrillar one. The SEM images of this system (Figure 5e) show the presence of small pores (ca. $500 \mathrm{~nm}$ ) in the polymeric matrix.

However, the formation of nanoscopic elongated fibrillar structures was observed in the hybrid gel $11 \mathrm{wt} . \%$, which shows a more homogeneous distribution (Figures $5 f$ and $5 \mathrm{~g}$ ) with fiber diameters of around $13 \mathrm{~nm}$, compatible with compound 1 (Figure 5a). Also, Figure 5h shows large interconnected pores of around $8 \mu \mathrm{m}$ in the $11 \mathrm{wt} . \%$ hydrogel.

Finally, a major change in hydrogel morphology was observed as the peptide was incorporated into the PEG polymer matrix, indicating that pore size formation is possible when increasing the amount of peptides in the system.

Microscopy images corroborate the results discussed so far. First, we observed that for hydrogels with higher amounts of peptide-silane, the presence of dispersed peptide fibers in the amorphous PEG structures favors stronger gel formation, as confirmed by rheology. Such microscopic images also reinforce the SAXS analysis model in this work, which shows two independent components in the hybrid hydrogel systems. Finally, rheological investigations were carried out to study the role of peptide motif $\mathbf{1}$ on the elastic modulus of the hydrogels. Figure S6 (SI section) shows that 


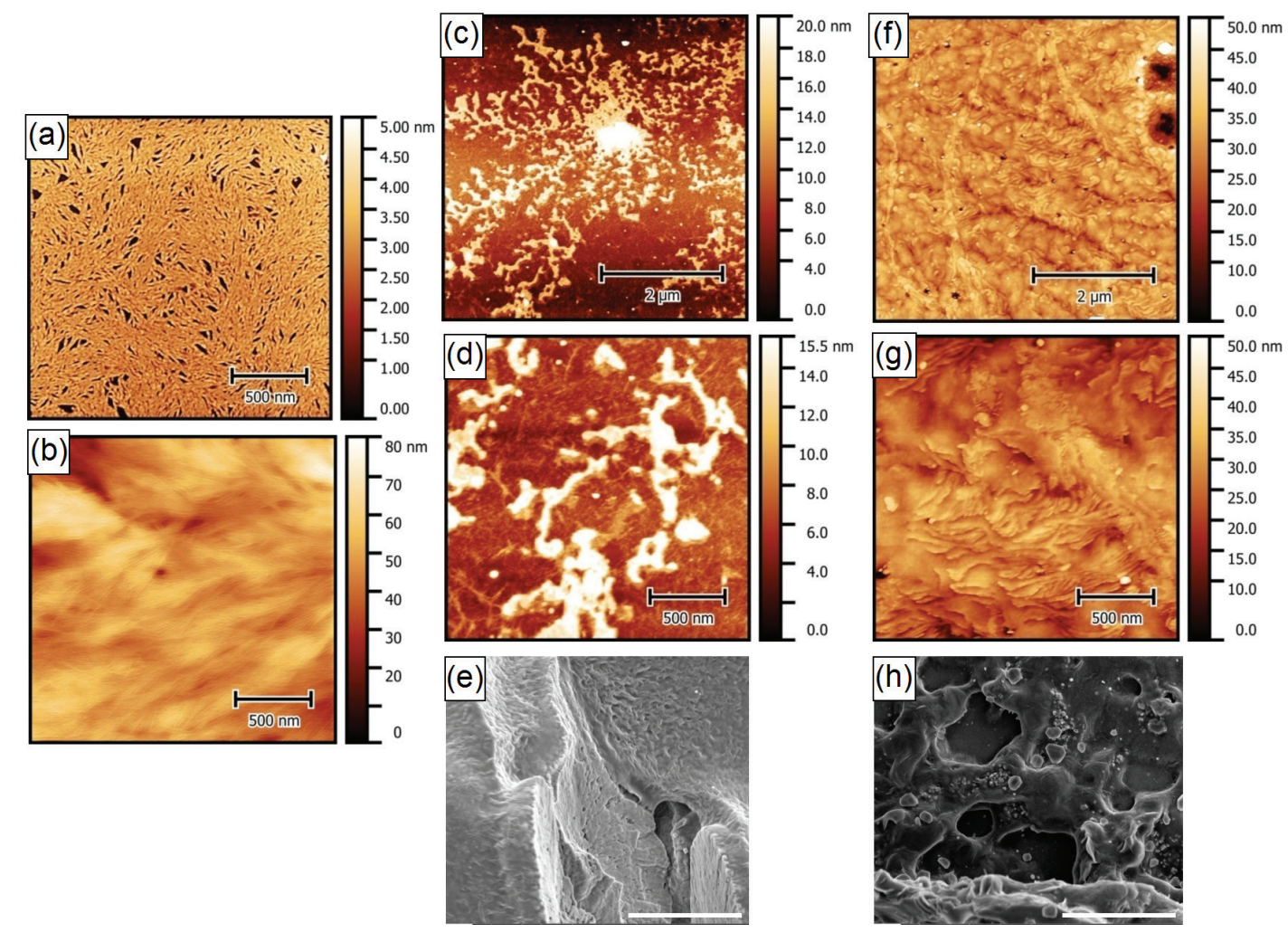

Figure 5. AFM images for (a) compound 1; (b) compound 2; (c-d) hydrogel 4.0 wt.\%; (f-g) hydrogel 11 wt.\%. SEM images (e) hydrogel 4.0 wt.\%; (h) hydrogel $11 \mathrm{wt} . \%$.

the $11 \mathrm{wt} . \%$ hydrogel presents thixotropic behavior, as the descending curve does not overlap the ascending curve. The sample presented strong resilience when the shear stress was removed, proving that the hydrogels obtained in this work can be used in the future as vehicles for topical drug delivery, in order to obtain a sustained drug release into the skin.

\section{Conclusions}

This work studies the formation of hybrid hydrogels from the sol-gel process by using $\beta$-amyloid-like peptides $[\mathrm{RF}]_{4}$ functionalized with alkoxysilanes, covalently bonded to the polyethylene glycol polymeric matrix. The successful one-step synthesis of this hybrid silylated amyloidogenic peptides and their respective hydrogels was achieved by the presence of typical bands of silane groups and corroborated by NMR, FTIR, and SAXS spectroscopies.

One of the advantages of this methodology is that the hydrogels' mechanical properties can be controlled by changing peptide concentration in the system, which increases the crosslinking density in these supramolecular polymeric materials. FTIR spectroscopy shows the secondary peptide structure conversion from anti-parallel to parallel $\beta$-sheets while rheologic analysis shows its correlation with the viscoelastic properties of the system, leading to the conclusion that when there is an increase in the number of $\beta$-sheets, there is a significant increase in the storage moduli.

SAXS analyses demonstrated that there was no change in the morphology of compound $\mathbf{1}$ when it was functionalized with silane group and glycine residues. They also proved that the peptide-polymer hybrid presents two independent scattering models, which adjusted the experimental data in the hybrid hydrogels.

AFM and SEM images revealed an interconnected porous morphology that corresponds to the intersection of polymer chains to form hydrogels, corroborating with rheology and SAXS data, indicating that the pore size can be controlled by increasing the amount of silane peptide in the polymeric networks. The rheology results demonstrated that the supramolecular hydrogels exhibit a shear thinning behavior. We also verified that the structure of the material recovered over time after shear deformation.

\section{Supplementary Information}

Supplementary information is available free of charge at http://jbcs.sbq.org.br as PDF file.

\section{Acknowledgments}

This work was supported by FAPESP (grant 
No. 2017/02317-2) and CNPq (grant No. 304389/2019-6). INCT in Bioanalytics (FAPESP grant No. 2014/50867-3 and CNPq grant No. 465389/2014-7) is kindly acknowledged for the grants. This study was also financed in part by the Coordenação de Aperfeiçoamento de Pessoal de Nível Superior, Brasil (CAPES), finance code 001. S. V. V. acknowledges CAPES for a doctoral fellowship. B. B. G. acknowledges FAPESP for a post-doctoral fellowship (project number 2018/05888-3). The staff at LNNano and LNLS are kindly recognized for their invaluable help and access to AFM (project number AFM-23070), SEM (project number SEM-24177) and SAXS Beamline (SAXS1-20180103) facilities. The authors are grateful to the Multiuser Central Facilities at UFABC.

\section{References}

1. Ulijn, R. V.; Bibi, N.; Jayawarna, V.; Thornton, P. D.; Todd, S. J.; Mart, R. J.; Smith, A. M.; Gough, J. E.; Mater. Today 2007, 10,40 .

2. Place, E. S.; Evans, N. D.; Stevens, M. M.; Nat. Mater. 2009, $8,457$.

3. Radvar, E.; Azevedo, H. S.; Macromol. Biosci. 2019, 19, 1800221.

4. Tibbitt, M. W.; Anseth, K. S.; Biotechnol. Bioeng. 2009, 103, 655.

5. Peppas, N. A.; Hilt, J. Z.; Khademhosseini, A.; Langer, R.; Adv. Mater. 2006, 18, 1345.

6. Zhang, S. G.; Nat. Biotechnol. 2003, 21, 1171.

7. Smith, D. K.; Adv. Mater. 2006, 18, 2773.

8. Otter, R.; Besenius, P.; Org. Biomol. Chem. 2019, 17, 6719.

9. Hamley, I. W.; Biomacromolecules 2014, 15, 1543.

10. Otter, R.; Henke, N. A.; Berac, C.; Bauer, T.; Barz, M.; Seiffert, S.; Besenius, P.; Macromol. Rapid Commun. 2018, 39, 1800459.

11. Castelletto, V.; Cheng, G.; Stain, C.; Connon, C. J.; Hamley, I. W.; Langmuir 2012, 28, 11599.

12. Hamley, I. W.; Ansari, A.; Castelletto, V.; Nuhn, H.; Rosler, A.; Klok, H. A.; Biomacromolecules 2005, 6, 1310.

13. Bibian, M.; Mangelschots, J.; Gardiner, J.; Waddington, L.; Acevedo, M. M. D.; de Geest, B. G.; Van Mele, B.; Madder, A.; Hoogenboom, R.; Ballet, S.; J. Mater. Chem. B 2015, 3, 759.

14. Hamley, I. W.; Chem. Rev. 2012, 112, 5147.

15. Domling, A.; Wang, W.; Wang, K.; Chem. Rev. 2012, 112, 3083.

16. Hamley, I. W.; Angew. Chem., Int. Ed. Engl. 2007, 46, 8128.

17. Knowles, T. P. J.; Mezzenga, R.; Adv. Mater. 2016, 28, 6546.

18. Cobo, I.; Li, M.; Sumerlin, B. S.; Perrier, S.; Nat. Mater. 2015,
14, 143 .

19. Echalier, C.; Pinese, C.; Garric, X.; Van Den Berghe, H.; Bilak, E. J.; Martinez, J.; Mehdi, A.; Subra, G.; Chem. Mater. 2016, 28,1261 .

20. Jebors, S.; Cecillon, S.; Faye, C.; Enjalbal, C.; Amblard, M.; Mehdi, A.; Subra, G.; Martinez, J.; J. Mater. Chem. 2013, 1, 6510 .

21. Echalier, C.; Jebors, S.; Laconde, G.; Brunel, L.; Verdie, P.; Causse, L.; Bethry, A.; Legrand, B.; Van Den Berghe, H.; Garric, X.; Noel, D.; Martinez, J.; Mehdi, A.; Subra, G.; Mater. Today 2017, 20, 59 .

22. Decandio, C. C.; Silva, E. R.; Hamley, I. W.; Castelletto, V.; Liberato, M. S.; Oliveira, V. X.; Oliveira, C. L. P.; Alves, W. A.; Langmuir 2015, 31, 4513.

23. Silva, E. R.; Listik, E.; Han, S. W.; Alves, W. A.; Soares, B. M.; Reza, M.; Ruokolainen, J.; Hamley, I. W.; Biophys. Chem. 2018, 233, 1 .

24. Pelin, J.; Gerbelli, B. B.; Soares, B. M.; Aguilar, A. M.; Alves, W. A.; Catal. Sci. Technol. 2019, 9, 4304.

25. Carpino, L. A.; Han, G. Y.; J. Am. Chem. Soc. 1970, 92, 5748.

26. Kaiser, E.; Colescott, R. L.; Bossinger, C. D.; Cook, P. I.; Anal. Biochem. 1970, 34, 595.

27. Wojdyr, M.; J. Appl. Crystallogr. 2010, 43, 1126.

28. Hammersley, A. P.; J. Appl. Crystallogr. 2016, 49, 646.

29. Oliveira, C. L. P.; Behrens, M. A.; Pedersen, J. S.; Erlacher, K.; Otzen, D.; J. Mol. Biol. 2009, 387, 147.

30. Behrendt, R.; White, P.; Offer, J.; J. Pept. Sci. 2016, $22,4$.

31. Gongora-Benitez, M.; Tulla-Puche, J.; Albericio, F.; ACS Comb. Sci. 2013, 15, 217.

32. Bechekh, K.; Ghaouar, N.; J. Macromol. Sci., Part B: Phys. 2014, 53, 391.

33. Padmanaban, R.; Venkatramanan, K.; Girivel, S.; Kasthuri, K.; Usharani, A.; Vellaichamy, R.; Springer Proc. Phys. 2017, 189, 723.

34. Frey, B. L.; Corn, R. M.; Anal. Chem. 1996, 68, 3187.

35. Eker, F.; Griebenow, K.; Schweitzer-Stenner, R.; Biochemistry 2004, 43, 6893.

36. Bergmann, A.; Fritz, G.; Glatter, O.; J. Appl. Crystallogr. 2000 , $33,1212$.

37. Glatter, O.; J. Appl. Crystallogr. 1977, 10, 415.

38. Teixeira, J.; J. Appl. Crystallogr. 1988, 21, 781.

39. Nečas, D.; Klapetek, P.; Cent. Eur. J. Phys. 2012, 10, 181.

Submitted: January 15, 2020

Published online: April 2, 2020 